\title{
Meeting Services and Networks in the Future Internet
}

\author{
Eduardo Santos ${ }^{1}$, Fabiola Pereira ${ }^{1}$, João Henrique Pereira ${ }^{2}$, \\ Luiz Cláudio Theodoro ${ }^{1}$, Pedro Rosa ${ }^{1}$, and Sergio Takeo Kofuji ${ }^{2}$ \\ 1 Federal University of Uberlândia, Brazil \\ eduardo@mestrado.ufu.br, fabfernandes@comp.ufu.br, lclaudio@feelt.ufu.br, \\ pedro@facom.ufu.br \\ 2 University of São Paulo, Brazil \\ joaohs@usp.br, kofuji@pad.lsi.usp.br
}

\begin{abstract}
This paper presents the researches for better integration between services and networks by simplifying the network layers structure and extending the ontology use. Through an ontological viewpoint, FINLAN (Fast Integration of Network Layers) was modeled to be able to deal with semantics in the network communication, cross-layers, as alternative to the TCP/IP protocol architecture. In this research area, this work shows how to integrate and collaborate with Future Internet researches, like the Autonomic Internet.
\end{abstract}

Keywords: Future Internet, Network Ontology, Post TCP/IP, Services

\section{Introduction}

In recent years it has been remarkable the Internet advancement in throughput and the development of different services and application features. Many of these are supported by the TCP/IP protocols architecture, however, the intermediate layers based on the protocols IP, TCP, UDP and SCTP were developed more than 30 years ago, when the Internet was used just for a limited number of hosts and with a few services support. Despite the development of the Internet and its wonderful flexibility and adaptability, there were no significant improvements in its Network and Transport layers, resulting in a communication gap between layers [7,8].

Integration of services and networks is an emerging key feature in the Future Internet and there are a lot of studies, proposals and discussions over questions related to a network able of supporting the current and Future Internet communication challenges. Some of these studies are related to: EFII, FIA, FIRE, FIND, GENI and other groups. Some of these groups are very expressive, for example FIRE, that includes discussions and projects like: ANA, AUTOI, BIONETS, CASCADAS, CHIANTI, ECODE, EFIPSANS, Euro-NF, Federica, HAGGLE, MOMENT, NADA, N4C, N-CRAVE, OneLab2, OPNEX, PERIMETER, PII, PSIRP, ResumeNet, Self-NET, SMART-Net, SmoothIT, TRILOGY, Vital++, WISEBED and 4WARD [2].

J. Domingue et al. (Eds.): Future Internet Assembly, LNCS 6656, pp. 339 350 2011.

(C) The Author(s). This article is published with open access at SpringerLink.com. 
Considering the possibilities for improvements in the current TCP/IP architecture with collaboration for the Future Internet, this work is focused in one alternative to the TCP/IP protocols, at layers 3 and 4, in one perspective to meet the service requirements in a simplified and optimized way, taking into account the real world service needs. This research also shows one proposal to improve the communication between services and networks with semantics, disseminating the power of the meaning across the network layers.

\section{Ontological Approach in FINLAN}

The FINLAN (Fast Integration of Network Layers), presented in [16], is a post-IP research which eliminates the Network and Transport layers, meeting services directly to the network lower layers. Thereby, the networks are prepared to meet the requirements of services in a flexible and optimized way. For example, the work in [6] shows how FINLAN can deal with the requirement of delivery guarantee, presenting its ability to service adaptability related to the applications needs and, in [1], it is presented the FINLAN implementation and experimental results compared with TCP/IP.

In this area of possibilities, in [5, 7, 10] our group discuss some fundamental aspects for the Post-IP research area and proposals to extend the use of ontology in computer networks to support the communication needs in a better way. Another aspect that can be placed in the context of the Future Internet is the use of ontology in networks. In current networks, the semantic communication generally is limited to the Application layer and this layer is restricted to sending meaning to the Network and Transport layers. Therefore, working with an ontological view over the Network and Transport layers has proved a promising object of research.

\subsection{Ontological Layers Representation}

The use of ontology at the intermediate layers permits the Internet Application layer better inform its needs to the intermediate layers. This increases the comprehension cross layers and contributes to get the upper and lower layers semantically closer. This helps the networks to improve the support to the communication needs, as the Application layer can inform, or request, requirements that the current TCP/IP layers 3 and 4 can not comprehend completely. Some of the communication needs that the lower layers can better support, by the ontology use in this work, are: Management, Mobility, QoE, QoS and Security.

This ontology at the intermediate layers is represented in FINLAN by the Net-Ontology and the DL-Ontology (Data Link) layers. The Net-Ontology layer has semantic communication, in OWL (Web Ontology Language), with its superior layer and the DL-Ontology layer. It is responsible to support the services needs of the superior layer. The DL-Ontology layer has semantic communication, also using OWL, with the superior and the Net-Ontology layers. It is responsible to support the Data Link communication to guarantee the correct delivery 
of data transfer between links. The main difference between these two layers is that the Net-Ontology layer is responsible to support service needs beyond simple data transfers. These layers, compared with the TCP/IP layers, are represented in Fig. 1, with examples of some Future Internet works that can be integrated with this approach at the intermediate layers.

TCP/IP

\begin{tabular}{|c|}
\hline Application \\
\hline Transport \\
\hline Network \\
\hline Data Link \\
\hline Physical \\
\hline
\end{tabular}

FINLAN

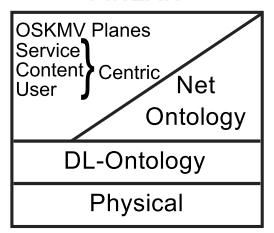

Fig. 1. TCP/IP and FINLAN Layers Comparison

From this layers comparison, some responsibilities of the actual TCP/IP Application, Transport and Network layers, are handled by the Net-Ontology layer, and some others by the Application layer. The applications, in FINLAN, inform their needs to the Net-Ontology layer using concepts. One application example is the encryption for security at the intermediate layer. In this example, in the actual TCP/IP protocols architecture, the layers 3 and 4 are not able to understand the security need in a context and its complexities usually must be controlled by the Application layer. However, in FINLAN, the Application layer can inform semantically this security need to the Net-Ontology layer. By this, the related complexities can be handled at the Net-Ontology layer level, instead of the Application layer level.

One layer level below, the difference between the TCP/IP Data Link and DLOntology layers is the semantic support, as the Data Link, in the TCP/IP, also does not support concepts. One application example is the services integration in heterogeneous environment to the devices mobility in $4 \mathrm{G}$ networks handovers, using the DOHand (Domain Ontology for Handover). In the experimental use of the DOHand, by Vanni in [17, the semantic possibilities for handover are reduced by the TCP/IP limitations. In this scenario, FINLAN approach contributes to the semantic communication between the DOHand and the DL-Ontology layer, for the handover in $4 \mathrm{G}$ networks.

In another application example, the FINLAN ontology can be used by the Management plane in OSKMV (Orchestration, Service Enablers, Knowledge, Management and Virtualisation planes), presented in [3], to better monitor the networks, as the semantic information can directly be handled by the NetOntology and DL-Ontology layers. Using the TCP/IP protocols architecture there are some limitations for the software-driven control network infrastructure, formed by the OSKMV, as the IP, TCP, UDP and SCTP protocols can not support some of the OSKMV needs directly in their protocols stack. These protocols generally just can send information at the data field and do not support semantic in their stacks. 
This possibility, to use ontology at the intermediate networks layers, can also contribute to the translations of the MBT (Model-based Translator) software package, by the use of the FINLAN formal representation in OWL. Similar considerations about contributions can be done at the Service, Content and User Centric approaches, when using the current TCP/IP layers 3 and 4, and not one Clean-Slate approach. Through the use of FINLAN ontology layers, explicit represented in OWL, the OSKMV planes and the Service, Content and User Centric works can have the benefit to inform their needs to the Net-Ontology and DL-Ontology layers and better approximate the upper and lower layers semantically. For this, the next section gives one example of the FINLAN ontology approach use and the section 2 expands the possibilities.

\subsection{FINLAN Ontology Example}

Discussions about the use of ontology languages in the interface between network layers, instead of protocols, are pertinent. However, they do sense by the service concept and service provider definitions defended by Vissers, long time ago, in his defense about important issues in the design of data communications protocols 18. Vissers' position also does sense for some current and Future Internet proposals by the separation of the internal complexities of each layer and exposing only the interfaces between them. In these concepts, the use of one ontology language also contributes to give a formal semantic power at the layers interface. This work uses OWL as formal language for this communication, as the OWL was adopted by a considerable number of initiatives and is a W3C standard. However, as the foundation is the ontology use at the intermediate layers, others formal ontology languages can be used.

One example of the FINLAN ontology use in the Future Internet research area is the possibility to support the AUTOI Functional Components communication with (and between) the network elements. So, the interactions between these components and their communication with the network intermediate layers can use OWL, instead of IP, UDP and TCP protocols. In a real integration, the migration of the end-user content service traffic presented in [14], section 4, for the Autonomic Management System (AMS) at the interaction migrateService(contentService), is understood by the FINLAN Net-Ontology layer with:

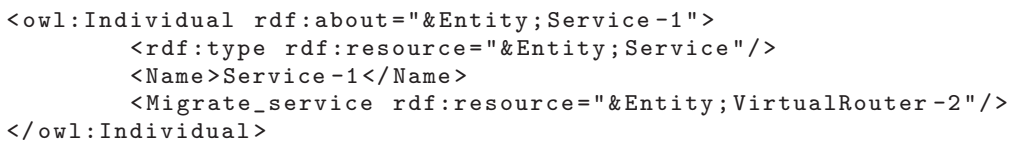

The Entity concept in the FINLAN ontology is one subclass of Thing (the OWL superclass) and the Service is one subclass of Entity.




This work shows how FINLAN can contribute with Future Internet researches (using AutoI as integration example) and it is not scope to describe the ontology foundation concepts and the implementations to enable the network communication without using the IP, TCP, UDP and SCTP protocols, as these studies and results are presented in some of our previous works [4 10, 16.

\section{Contributions to the Future Internet Works}

The FINLAN project has adherence with some current efforts in the Future Internet research area, and the representation example above shows that the ontology cross layers can, naturally, be integrated with other initiatives. Its use is possible in a wide range of scenarios and is possible to visualize some characteristics and advantages. Attempting to the alignment with some Future Internet groups proposals, the next section extends possible collaborations that may be implemented in an integrated way with some works.

For better understanding, Fig. 2 illustrates an overview of the basic concepts of FINLAN ontology. The main classes are defined as follows:

- DIS (Domain ID System): is responsible for IDs resolution in FINLAN. Therefore, it manages the network's addressing;

- Entity: the class that represents all that can establish communication. For example: a service, a content, a network element and even a cloud computing;

- ID: the unique identifier of each entity;

- Layer: class representing a layer in FINLAN. It contains four sub-classes (Application, Net-Ontology, DL-Ontology and Physical) relating to the FINLAN layer structure, as illustrated in Fig. 1]

- Necessity: represents the requirements that an entity has during communication. For instance: delivery guarantee, QoS, security and others.

\subsection{Collaboration to the AutoI Planes}

One of the Autonomic Internet project expectations is to support the needs of virtual infrastructure management to obtain self-management of virtual resources which can cover heterogeneous networks and services like mobility, reliability, security and QoS. The FINLAN project can contribute in its challenges, some described in 1, 3, 12, to have the intermediate layers support for selfawareness, self-network and self-service knowledge.

Virtual Resources Management: The FINLAN ontology supports the network communication used by the AutoI vCPI (Virtual Component Programming Interface) [13, allowing a localized monitoring and management of the virtual resources. By this, the FINLAN ontology layers can comprehend communication needs as the instantiation, remotion and modification of virtual resources.

To support the information monitoring which is essential to the functions of self-performance and fault management, the FINLAN, through its ontology 




Fig. 2. Overview of FINLAN Ontology

design, can comprehend and do actions in information like: use of the CPU, memory assignment, packets lost and others. The invocation of the methods can be done by the AMSs, DOCs (Distributed Orchestration Component) or ANPI (Autonomic Network Programming Interface) and one OWL sample code for this communication is showed bellow:

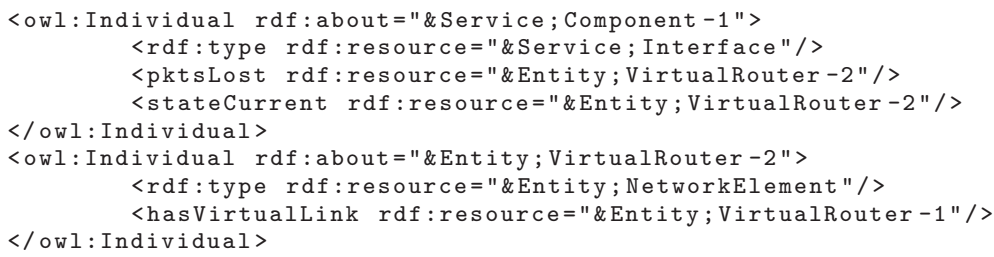

In the FINLAN ontology, an Interface is a subclass of the Service Entity and two network elements, like virtual routers, can interact between them through the property hasVirtualLink.

\section{Collect, Dissemination and Context Information Processing: FINLAN} allows to create the Net-Ontology interface with AutoI to support the contextaware control functions for the self-management and adaptation in the CISP (Context Information Services Platform) needs. The context information in the FINLAN layers can act as an intermediate unity with its own semantic to reduce 
the number of interactions between the context sources and the context clients, diminishing the network effort in some cases.

The network context, for example, can interact with network elements and services according to the ontology concepts in the following code, where the context NetContext-1 is in the network element VirtualRouter-2 and the service Service-1 has information to the network context NetContext-1.

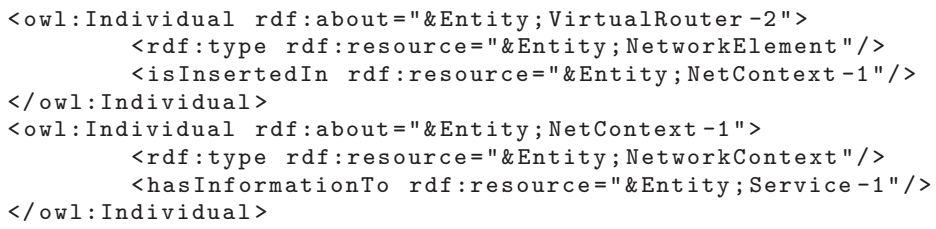

Management of Active Sessions: The AutoI open source implements a scalable and modular architecture to the deployment, control and management of active sessions used by virtual entities. It consists of one active element and the forwarding engine like a router [15].

Its integration with FINLAN can act in some components, like the Diverter, the Session Broker and the Virtualisation Broker. There are many others but these are essentials. As the communication between the AutoI modules is done through UDP transactions or TCP connections, FINLAN can collaborate in this scenario, facilitating the effort for the AutoI in the implementation of the network semantic support for them. The representation of the Session Broker communicating with a service is showed in the example below:

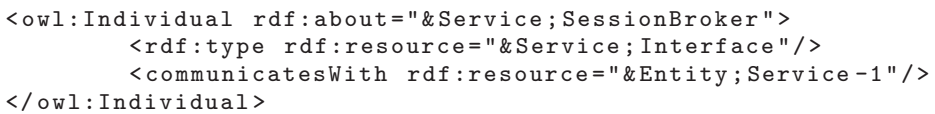

The representation of the FINLAN project collaboration with AutoI is showed in Fig. 3, extended from the AutoI planes figure presented in 3.

\subsection{Collaboration to the RESERVOIR Service Provider}

The FINLAN project does not intend to conflict with the RESERVOIR proposals, as RESERVOIR also has the objective to supply an architecture and the reference implementation to a service oriented infrastructure. On the other hand, the FINLAN project aims to contribute with researches to build new technologies to replace the traditional TCP/IP layers 2,3 and 4, giving semantic features to the network intermediate layers. In this proposal, is possible to use the RESERVOIR manifest formally defined in OWL to the contract and the SLA between the service provider and the infrastructure provider. As the manifest has specifications and rules well defined it facilitates the ontology creation to support them in FINLAN, and to create its individuals.

Specific information about components, as maximum and minimum, requisites for an instance (memory size, storage pool size, number of virtual CPUs, 




Fig. 3. FINLAN Collaboration with AutoI Planes

number of network interfaces and its bandwidths) are aspects to be worked in an effective collaboration. For this, the FINLAN ontology supports, for example, services that communicates with the ServiceCloud Entity, which has the need of the information stored in the manifest requirement. The ontology concept for this example is:



\subsection{Collaboration to the Complexity Reduction for $\{$ User, Service, Content\}-Centric Approaches}

This work can collaborate to reduce the complexity of the network use by the user, service and content centric projects, as the ontology can offer better comprehension for the networks. About the proposals for a Clean-Slate solution this work also gives collaboration, by the OWL experiments at the intermediate network layers and the cross layers communication.

In the researches of service-centric, the FINLAN is placed to the level of the network elements and generates semantic support and answers to the higher layer needs. For example, to handle requests for services related to bandwidth, storage, encryption, location, indexing and others.

Related to the content-centric it is presented in [19] the difficulties of the current networks to support the objects concept. In order to reduce the complexity in this implementation it was brought to FINLAN the concept of content object providing the formalization to one Clean-Slate approach. 
In this proposal, the objects Media, Rules, Behaviour, Relations and Characteristics, components of ALLOA (Autonomic Layer-Less Object Architecture), can use the FINLAN to easily interact with the network elements, simplifying the communication process with the lower layers.

Through the FINLAN Net-Ontology layer, requirements such as QoS and Security, can be requested to the network, making the \{user, service, content $\}$ centric approaches simpler, as shown in the sample code below:

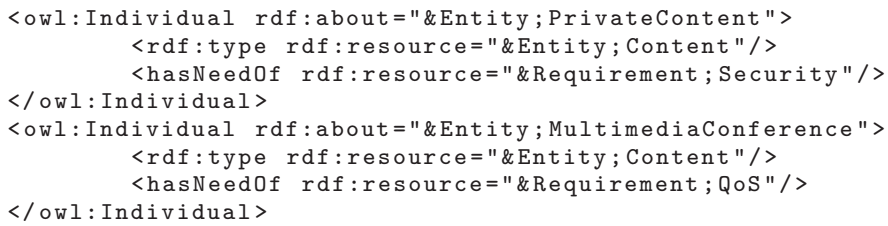

\section{$3 \quad$ Integration between Services and Networks}

This section describes how to integrate this project in collaboration with others Future Internet works, continuing the examples with the AutoI integration. As the AutoI project has been fulfilling its purposes, it is observed that the evolution of TCP/IP layers to increase the networks communication possibilities, is a growing need and can not be disregarded to the future of the Internet infrastructure.

AutoI modules connections are performed in well defined form using connection handlers or similar classes that uses TCP/IP sockets. The AutoI integration with networks using semantics, instead of TCP/IP protocols, can be done using the FINLAN library. The benefit of this integration for the AutoI Orchestration plane is the use of the ontology at the intermediate layers to support the semantic needs to orchestrate the negotiation between the AMSs and the communications with the network elements. Other benefits are the possibility for the DOC to request different needs to the network layer.

\subsection{Integration Using FINLAN Library}

The FINLAN library uses raw sockets for sending and receiving packets directly between the data link and the Application layer. The implementation of the FINLAN proposal, supporting ontology, incorporates the concepts discussed in [16] and [6] and extends this functionality as it allows the understanding of the needs for establishing communication through the ontological model adopted.

For the example described in 1.2, to the service migration, the ANPI receives the migrateService (contentService) from the AMS. Based on the AutoI Java open source, in the ANPI demo, the ANPISDD class is prepared to use the IP and TCP (port 43702) protocols. So the SDD (Service Deployment Daemon) runs using the traditional TCP/IP stack, as in the following sample code extracted from the ANPISDD.java code. 


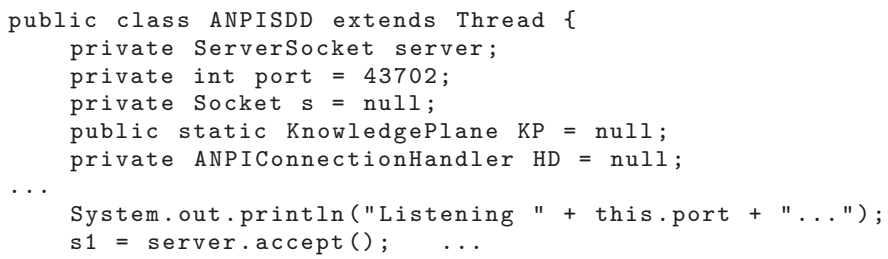

With the use of the FINLAN library this communication can be done replacing the IP and TCP protocols with the FINLAN representation using OWL over raw sockets for the Net-Ontology and DL-ontology layers presented in Fig. 1. This expands the semantic possibilities for the AutoI planes, through the intermediate layers of the networks in the Future Internet, for the communication between the Service Enabler plane and the Management/Knowledge plane implemented by the AMS.

From a practical way, the FINLAN library implementation occurs through the development of Net-Ontology and DL-Ontology layers, which depend on the design of OWL concepts of the FINLAN proposal, illustrated in Fig. 2.

Fig. 4 shows how the Net-Ontology and DL-Ontology layers relate with the developed ontology, Services and Physical layers of the network. These layers are based on the formalization of the FINLAN concepts. This formalization was modeled in Protégé, also used to generate the OWL, by the OWL API (version 3.0.0.1451). In this figure, the arrows show that the concepts in OWL are loaded into Net-Ontology and DL-Ontology layers allowing the semantic communication and network behavior control.

From the OWL concepts, the Net-Ontology layer is skillful to receive requests for applications, which are transmitted via messages containing OWL tags, and the needs of the data flow that will start. With the understanding of application needs, the Net-Ontology layer, sends to the DL-Ontology layer another OWL object with the requirements of data communication as a way of addressing, for example. After the definitions of application requirements, the communication

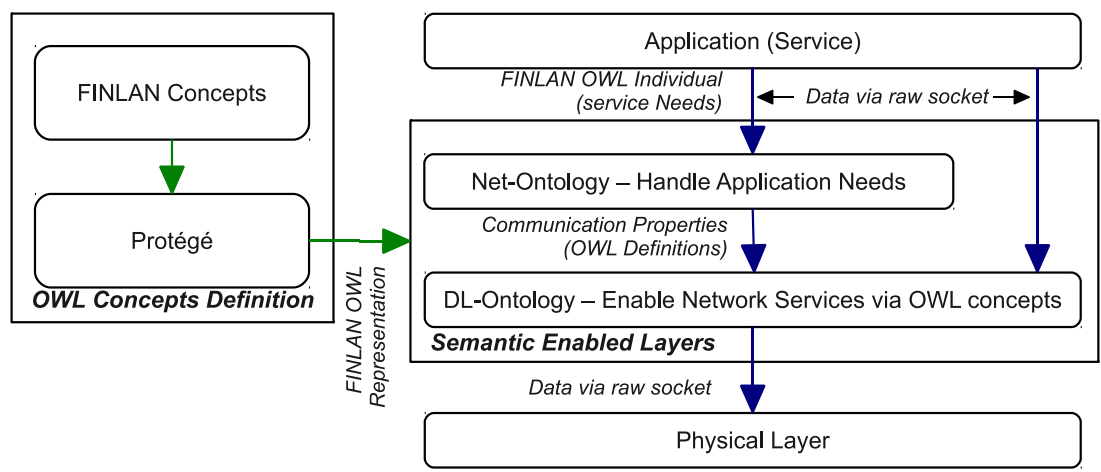

Fig. 4. Overview of FINLAN Library Implementation 
is ready to be established, and the data is sent through the layers also using raw sockets.

At the current stage of development the implementation of FINLAN library is made in application level. Nevertheless, the future intentions are to implement the FINLAN ontology in Linux operating system kernel level, allowing the facilities in its use in different programming languages, since the methods proposed would be available at the operating system level.

\section{Conclusions}

This paper has presented the FINLAN ontology works in a collaboration perspective with some Future Internet projects. We have proposed to better meeting of services and networks by approaching services semantically to the network structure. It was showed how to integrate FINLAN with Future Internet projects, taking AutoI as example, and how the ontological approach can be applied to Future Internet works like monitoring and content-centric Internet.

Future work will implement the FINLAN ontology at the Linux kernel level and run performance and scalability experiments with different Future Internet projects open implementations. Further work also will do the extension of the scope of the ontological representation, by modeling the behavior of FINLAN to support requirements in contribution with different Future Internet projects.

We strongly believe that meeting services and networks through the reduction of network layers and, consequently, through the decreasing of users, services and content complexity is a possible way to achieve flexibility in future networks. Moreover, we expect that ontological approaches can help to build a Future Internet with its real challenges, requirements and new paradigms.

Acknowledgment. This work is a result of conceptual discussions and researches of all members of the FINLAN group. The authors would like to acknowledge the implementations and philosophical talks with this group. Also to thank the efforts to gather on the state-of-the-art of the Future Internet.

Open Access. This article is distributed under the terms of the Creative Commons Attribution Noncommercial License which permits any noncommercial use, distribution, and reproduction in any medium, provided the original author(s) and source are credited.

\section{References}

[1] Clayman, S., Galis, A., Chapman, C., Toffetti, G., Rodero-Merino, L., Vaquero, L.M., et al.: Monitoring Service Clouds in the Future Internet. In: Towards the Future Internet - Emerging Trends from European Research, p. 115 (2010)

[2] FIRE: FIRE White Paper (Aug. 2009), http://www.ict-fireworks.eu/ fileadmin/documents/FIRE_White_Paper_2009_v3.1.pdf 
[3] Galis, A., Denazis, S., Bassi, A., Giacomin, P., Berl, A., A., Fischer, o.: Management Architecture and Systems for Future Internet. In: Towards the Future Internet - A European Research Perspective, p. 112 (2009)

[4] Malva, G.R., Dias, E.C., Oliveira, B.C., Pereira, J.H.S., Kofuji, S.T., Rosa, P.F.: Implementação do Protocolo FINLAN. In: 8th International Information and Telecommunication Technologies Symposium (2009)

[5] Pereira, F.S.F., Santos, E.S., Pereira, J.H.S., Rosa, P.F., Kofuji, S.T.: Proposal for Hybrid Communication in Local Networks. In: 8th International Information and Telecommunication Technologies Symposium (2009)

[6] Pereira, F.S.F., Santos, E.S., Pereira, J.H.S., Rosa, P.F., Kofuji, S.T.: FINLAN Packet Delivery Proposal in a Next Generation Internet. In: IEEE International Conference on Networking and Services, p. 32 (2010)

[7] Pereira, J.H.S., Kofuji, S.T., Rosa, P.F.: Distributed Systems Ontology. In: IEEE/IFIP New Technologies, Mobility and Security Conference (2009)

[8] Pereira, J.H.S., Kofuji, S.T., Rosa, P.F.: Horizontal Address Ontology in Internet Architecture. In: IEEE/IFIP New Technologies, Mobility and Security Conference (2009)

[9] Pereira, J.H.S., Kofuji, S.T., Rosa, P.F.: Horizontal Addressing by Title in a Next Generation Internet. In: IEEE International Conference on Networking and Services, p. 7 (2010)

[10] Pereira, J.H.S., Pereira, F.S.F., Santos, E.S., Rosa, P.F., Kofuji, S.T.: Horizontal Address by Title in the Internet Architecture. In: 8th International Information and Telecommunication Technologies Symposium (2009)

[11] Pereira, J.H.S., Santos, E.S., Pereira, F.S.F., Rosa, P.F., Kofuji, S.T.: Layers Optimization Proposal in a Post-IP Network. International Journal On Advances in Networks and Services, in Press (2011)

[12] Rochwerger, B., Galis, A., Breitgand, D., Levy, E., Cáceres, J., Llorente, I., Wolfsthal, Y., et al.: Design for Future Internet Service Infrastructures. In: Towards the Future Internet - A European Research Perspective, p. 227 (2009)

[13] Rubio-Loyola, J., Astorga, A., Serrat, J., Chai, W.K., Mamatas, L., Galis, A., Clayman, S., Cheniour, A., Lefevre, L., et al.: Platforms and Software Systems for an Autonomic Internet. In: IEEE Global Communications Conference (2010)

[14] Rubio-Loyola, J., Astorga, A., Serrat, J., Lefevre, L., Cheniour, A., Muldowney, D., Davy, S., Galis, A., Mamatas, L., Clayman, S., Macedo, D., et al.: Manageability of Future Internet Virtual Networks from a Practical Viewpoint. In: Towards the Future Internet - Emerging Trends from European Research, p. 105 (2010)

[15] Rubio-Loyola, J., Serrat, J., Astorga, A., Chai, W.K., Galis, A., Clayman, S., Mamatas, L., Abid, M., Koumoutsos, G.: et al.: Autonomic Internet Framework Deliverable D6.3. Final Results of the AutonomicI Approach. AutoI Project (2010)

[16] Santos, E.S., Pereira, F.S.F., Pereira, J.H.S., Rosa, P.F., Kofuji, S.T.: Optimization Proposal for Communication Structure in Local Networks. In: IEEE International Conference on Networking and Services, p. 18 (2010)

[17] Vanni, R.M.P.: Integração de Serviços em Ambientes Heterogêneos: uso de Semântica para Comunicação Entre Entidades em Mudanças de Contexto. Ph.D. thesis, University of São Paulo - USP (2009)

[18] Vissers, C.A., Logrippo, L.: The Importance of the Service Concept in the Design of Data Communications Protocols. Proceedings of the IFIP WG6 1, 3 (1986)

[19] Zahariadis, T., Daras, P., Bouwen, J., Niebert, N., Griffin, D., Alvarez, F., Camarillo, G.: Towards a Content-Centric Internet. In: Towards the Future Internet - Emerging Trends from European Research, p. 227 (2010) 